\title{
OPTICAL AND ELECTRICAL PROPERTIES OF UNDOPED MICROCRYSTALLINE SILICON DEPOSITED BY THE VHF-GD WITH DIFFERENT DILUTIONS OF SILANE IN HYDROGEN
}

N. BECK*, P. TORRES*, J. FRIC**, Z. REMEŠ**, A. PORUBA**, HA STUCHLÍKOVÁ**, A. FEJFAR**, N. WYRSCH*, M. VANĚČEK**, J. KOČKA**, A. SHAH*

* Institut de Microtechnique, Université de Neuchâtel, Rue A. L. Breguet 2, 2000 Neuchâtel, Switzerland

** Institute of Physics, Academy of Sciences of the Czech Republic, Cukrovarnická 10, 16200 Praha 6, Czech Republic

\section{ABSTRACT}

We show that the optical and electrical properties of microcrystalline silicon $(\mu \mathrm{c}-\mathrm{Si}: \mathrm{H})$ deposited by the VHF-GD technique at $110 \mathrm{MHz}$ can considerably be tuned by changing the dilution ratio of silane to hydrogen.

With increasing silane dilution we observe enhanced optical absorption for energies below $2 \mathrm{eV}$ due to the transition of the material from amorphous / microcrystalline mixture to a pure microcrystalline phase. Simultaneously, the light scattering and the defect absorption increases. Strong dilution also promotes the incorporation of impurities into the material, leading to a pronounced extrinsic behaviour as seen from the decrease of the activiation energy of the electrical conductivity.

The electrical properties were investigated in the dark by the Time of Flight technique. We measured drift mobilities at room temperature which slightly increase with dilution, reaching values of $3 \mathrm{~cm}^{2} / \mathrm{Vs}$ for electrons and $1.2 \mathrm{~cm}^{2} / \mathrm{Vs}$ for holes. The ratio between electron and hole drift mobilities is found to be around 2 for all samples studied, similar to that of crystalline silicon.

Furthermore, post-transient Time of Flight measurements revealed detrimental electron deep traps in low dilution material.

\section{INTRODUCTION}

With an enhanced optical absorption in the infrared region together with a low sub-bandgap absorption, the microcrystalline silicon produced by the VHF-GD deposition technique is a promising material for solar cell applications [1]. The absorption properties of such material is almost "crystalline-like" [2], but can also be tuned by changing the deposition parameters [3].

On the other hand, only very little is known, so far, about electrical transport in $\mu \mathrm{c}-\mathrm{Si}: \mathrm{H}$; the collection mechanism for carriers in corresponding $\mathrm{p}-\mathrm{i}-\mathrm{n}$ cells is therefore still unclear. Entirely microcrystalline p-i-n solar cells with thicknesses of up to $3.6 \mu \mathrm{m}$ [4] have been shown to collect almost the totality of photo-generated carriers even though coplanar transport (photoconductivity and ambipolar diffusion length) is only slightly higher in such $\mu \mathrm{c}-\mathrm{Si}: \mathrm{H}$ layers compared to amorphous silicon a-Si:H [5].

With the aim to gain more insight into absorption and transport mechanisms of microcrystalline silicon, we produced a series of $\mu \mathrm{c}-\mathrm{Si}: \mathrm{H}$ layers using different dilution ratios of silane in hydrogen for deposition. This enabled us to create materials with very different qualities, ranging from purely amorphous to highly microcrystalline material. The optical absorption measurements as well as the transport properties parallel to the growth direction reveal important changes within this series, mainly with respect to the density and activity of deep defects, which will be presented and discussed in the present paper. 


\section{EXPERIMENTAL}

The samples were deposited by the VHF-GD deposition technique at a plasma excitation frequency of $110 \mathrm{MHz}$ using a gas purifier [6]. Thereby the ratio of silane flow over total gas flow $\left[\mathrm{SiH}_{4}\right] /\left[\mathrm{SiH}_{4}+\mathrm{H}_{2}\right]$ was varied between $1.25 \%$ and $7.5 \%$. The sample deposited at $7.5 \%$ dilution is amorphous, all the others are microcrystalline. With deposition rates ranging from less than $0.7 \AA / \mathrm{s}$ (1.25\% dilution) to $3.5 \AA / \mathrm{s}$ (7.5\% dilution) layer thicknesses between 1.6 and $1.9 \mu \mathrm{m}$ were obtained. All films were deposited either on uncoated glass (Schott AF 45) or on chromium-coated glass (for Time of Flight). Semitransparent, small size $\left(\sim 2 \mathrm{~mm}^{2}\right.$ area) chromium top contacts were deposited for TOF samples, coplanar aluminium contacts with a gap of $0.5 \mathrm{~mm}$ were used for dark conductivity and CPM measurements.

The optical absorption of the materials was determined by Transmission/Reflection spectroscopy, by Constant Photocurrent Method (CPM) and by Photothermal Deflection Spectroscopy (PDS). The transport properties were studied by dark conductivity measurements at temperatures ranging from $25^{\circ} \mathrm{C}$ to $180^{\circ} \mathrm{C}$. Time of Flight (TOF) measurements in both shorttime and post transient mode were performed respecting the guidelines for TOF measurements on $\mu \mathrm{c}-\mathrm{Si}: \mathrm{H}$ presented in [7].

X-ray diffraction measurements on all microcrystalline samples presented here show a preferential growth in the $<220>$ direction. A medium grain size of around $200 \AA$ and columnar shape of the grains has already been found in the past for other samples produced using similar deposition conditions [1].

\section{RESULTS}

\section{Optical properties}

It has already been shown [3] that the transition from microcrystalline to amorphous material is abrupt and typically takes place around $7.5 \%$ dilution for the deposition conditions used here. The optical absorption spectra measured on the present series (represented in figure 1) confirms the drastic change of optical absorption with a variation of the dilution ratio. Thereby, three distinct effects can be observed: the

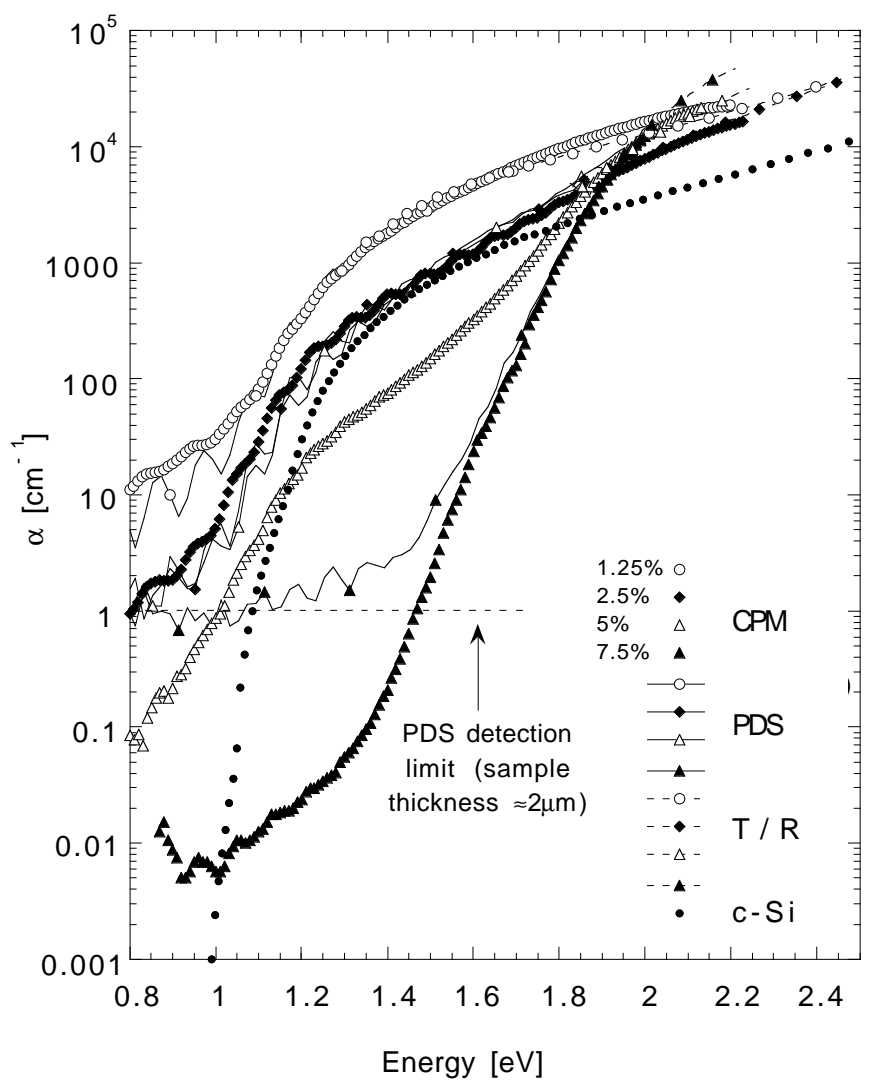

Fig. 1 Optical absorption coefficient for the $110 \mathrm{MHz}$ dilution series determined by PDS, CPM and Transmission / Reflection measurements: the transition from amorphous to microcrystalline silicon takes place at around $7.5 \%$ dilution. Crystalline silicon data [8] is also plotted for comparison. Note the difference between the CPM and PDS spectra for the 5\% sample, discussed in the text. 
transition from a-Si:H to $\mu \mathrm{c}-\mathrm{Si}: \mathrm{H}$, scattering and the effect of mixed-phase material.

First, the main phenomenon observed is of course the transition from non-direct absorption in amorphous material with a Tauc gap around $1.7 \mathrm{eV}$ to the optical absorption in the crystalline material with an indirect gap of about $1.1 \mathrm{eV}$. However, there are also significant changes in the absorption curve within the microcrystalline range of deposition conditions. As a second effect, for the sample with the highest dilution, further enhancement of optical absorption by scattering is apparent. We measured the scattered part of the total reflected light (and thus also of the penetrating light) and observed an increase from about $8 \%$ at $650 \mathrm{~nm}(5 \%$ dilution sample) to more than $50 \%$ for the highly diluted sample $(1.25 \%$

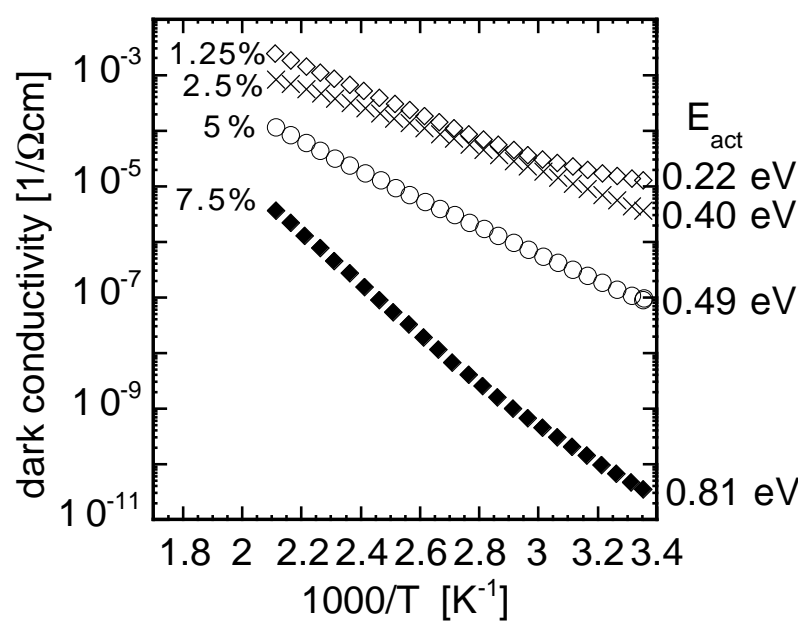

Fig. 2 Measured dark conductivities as a function of temperature for different dilutions. The sample with $7.5 \%$ dilution is amorphous, all the others are microcrystalline. The activation energies were determined at room temperature.

dilution). As the scattering events result in a longer effective optical path of the photons, an enhanced apparent absorption is observed [9].

Third, the difference between CPM and PDS deduced optical absorption (as clearly seen in the sample with 5\% dilution) is due to the mixture of amorphous and crystalline phase prevalent in this particular case. PDS sees the true optical absoprtion, whereas CPM curve can be deconvoluted into two contributions: now, above approx. $1.7 \mathrm{eV}$ the transport in amorphous tissue with higher photosensitivity gives an additional contribution to the "apparent optical absorption coefficient". Because of the calibration procedure used this results in an artificial shift of " $\alpha$ " to lower values for photon energies below $1.7 \mathrm{eV}$.

When looking at the subbandgap optical absorption (below $1 \mathrm{eV}$ ), PDS cannot see the differences between the samples (due to its "detection limit"). On the other hand, CPM absorption, which is not masked by the substrate, interface and surface absorption, can detect the changes in the defect-connected absorption: the subbandgap absorption connected to defects is found to increase with increasing dilution ratios. This can indicate an increased number of dangling bonds, unsaturated by hydrogen, at the grain boundaries [3]. The possible role of oxygen impurities on the optical spectrum has not yet been studied, experiments are under preparation. However, post-transient TOF measurements performed on the same samples show a different trend. This apparent inconsistency will be addressed in more detail in the following section.

\section{Electrical properties}

Even though all layers were produced using a gas purifier in order to prevent the incorporation of impurities [6], the microcrystalline materials deposited with high dilutions show a pronounced extrinsic behaviour (Fig. 2) as is usually obtained in the presence of [O] contaminants. The measured dark conductivities vary from $1 \times 10^{-7} 1 / \Omega \mathrm{cm}$ (typical for "truly" intrinsic $\mu \mathrm{c}-\mathrm{Si}: \mathrm{H})$ to $1.3 \times 10^{-5} 1 / \Omega \mathrm{cm}$ with activation energies ranging from $0.49 \mathrm{eV}$ to $0.22 \mathrm{eV}$. In fact, the low deposition rates of the highly diluted $\mu \mathrm{c}-\mathrm{Si}: \mathrm{H}(0.67 \AA / \mathrm{s}$ for $1.25 \%$ compared to $2.2 \AA / \mathrm{s}$ for $5 \%$ dilution) can possibly promote the incorporation of oxygen as a donor impurity in the material and cause the observed shift of the Fermi level towards the conduction band edge. The layer deposited with $7.5 \%$ dilution shows values of 
dark conductivity and activation energy that are typical for intrinsic amorphous material (Fermi level close to midgap).

The characterisation of the carrier transport in the dark was made by the Time of Flight technique (TOF). Thereby, mainly two features designated this method as appropriate to study the carrier transport in our layers: first, the transport of electrons and holes can be measured independently and secondly the transport can be monitored in the growth direction of the layer, in a configuration close to that of a $\mathrm{p}-\mathrm{i}-\mathrm{n}$ cell. The results obtained for drift mobilities $\left(\mu^{\mathrm{D}}\right)$ at room temperature are presented in figure 3. Even though the transport properties vary less drastically than the optical properties, some of the observed features are remarkable. First of all, the drift mobilities clearly increase from 1 to $3 \mathrm{~cm}^{2} / \mathrm{Vs}$ (electrons) and from 0.4 to $1.2 \mathrm{~cm}^{2} / \mathrm{Vs}$ (holes) with increasing dilution at room temperature. Furthermore, in all measured $\mu \mathrm{c}-\mathrm{Si}: \mathrm{H}$ layers the drift mobilities for electrons are about twice the hole drift mobilities; this ratio is in clear contrast with the much higher ratio observed for a-Si:H $\left(\mu \mathrm{D}_{\mathrm{e}} / \mu^{\mathrm{D}} \mathrm{h} \approx 100\right)$. This observation strongly indicates that the drift mobility of carriers in $\mu \mathrm{c}-\mathrm{Si}: \mathrm{H}$ is not governed mainly by the amorphous tissue, as here band tail trapping would give rise to the typical strong mobility asymmetry. Thus, other mechanisms related to grain boundaries and/or scattering must be taken into account.

The measurements of drift mobilities of electrons and holes as a function of temperature $(300-380 \mathrm{~K})$ are plotted in figure 3 . Whereas the drift mobility $\mu^{\mathrm{D}}$ of electrons significantly decreases with increasing temperature for all microcrystalline samples, the opposite is observed for holes (except for the $1.25 \%$ dilution sample). The activation energies for hole mobilities are low, $59 \mathrm{meV}$ (5\% dilution) and $21 \mathrm{meV}$ (2.5\% dilution), respectively. Thereby, numerous processes can influence the drift mobility with changing temperature: thermoionic emission of carriers over barriers [10], scattering effects (which can reduce the mobility at high temperatures), but also deep trapping and tunneling may be involved. Thus, at this stage it is not possible to discriminate the dominant transport process in our $\mu \mathrm{c}-\mathrm{Si}: \mathrm{H}$ material and more extensive measurements will be necessary here.

Whereas short-time TOF monitors the transit of the photogenerated carrier package through the layer, the extension of the TOF experiment to a much longer time scale (post-transient TOF [11], [12]) gives information about the density of states (DOS) in the gap. Thereby, electrons and holes which were deep-trapped during transit are thermally reemitted at longer times and their collection becomes a direct fingerprint of the DOS. In our microcrystalline material the post transient currents from holes are featureless and no particular deep trap states for holes can be observed. On the other side, for electron transients, very pronounced detrapping at times around $10^{-3}-10^{-4} \mathrm{~s}$ appears for low-dilution samples (see Fig. $4 \mathrm{~b}$ and c), indicating thus the presence of efficient electron traps in the middle of the gap (about $0.5 \mathrm{eV}$
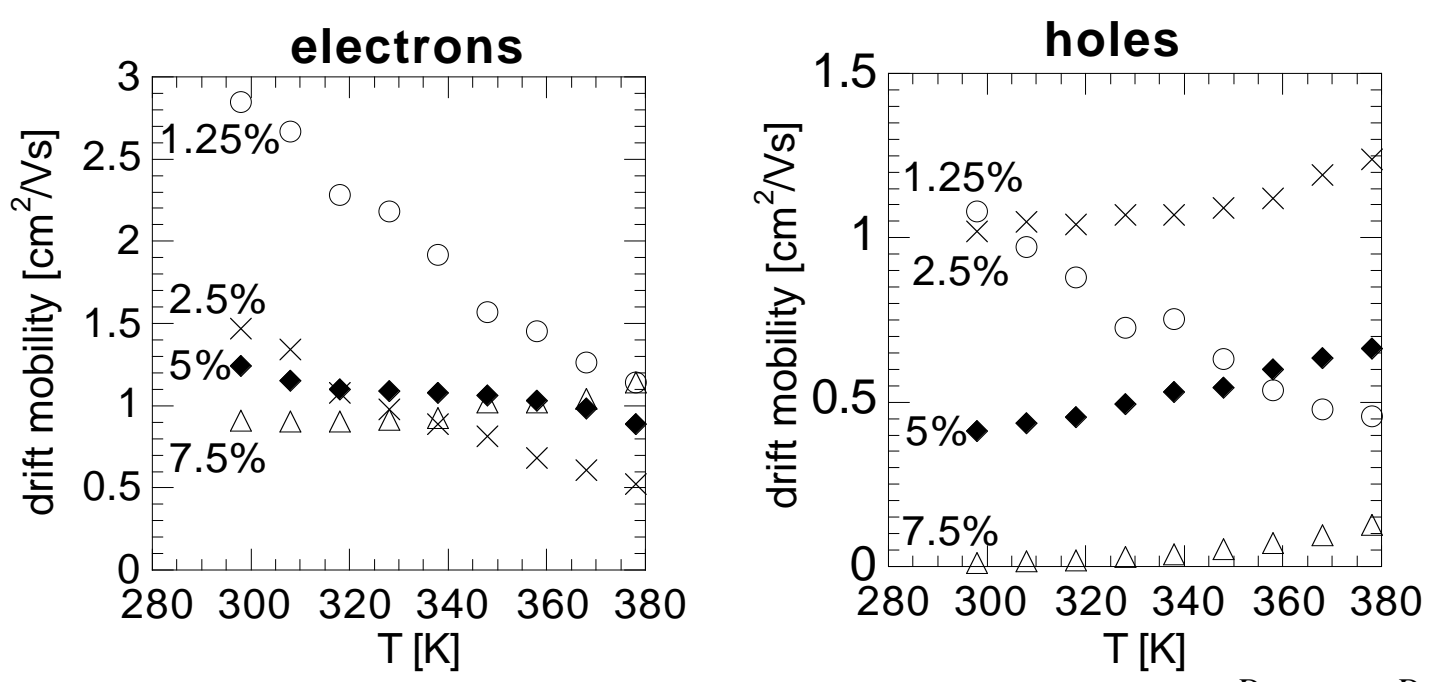

Fig. 3 Temperature dependency of electron and hole drift mobilities $\mu^{D_{e}}$ and $\mu^{D_{h}}$ as determined by Time of Flight. 

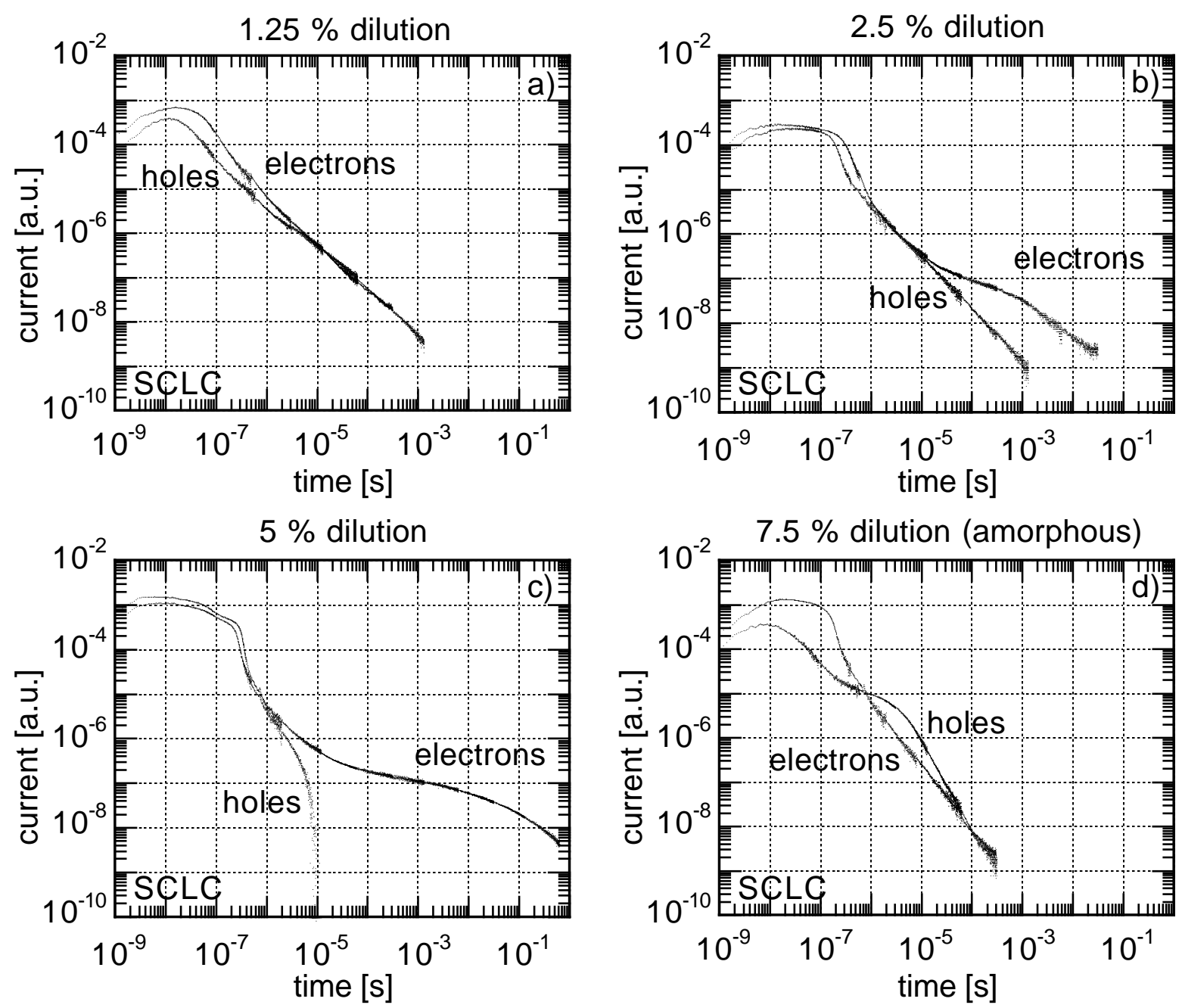

Fig. 4 Time of Flight post transient currents (in space charge limited mode SCLC) for electrons and holes: the low dilution microcrystalline samples (fig.4c, but also b) show a pronounced detrapping current for electrons, indicating the presence of a detrimental type of electron deep trap in this material. Note that the characteristic time in the SCLC mode is the extraction time which is different from the transit time used for drift mobility determination.

deep). As these traps are only active for electrons, we attribute them to positively charged defects at the grain boundaries. However, it is not clear at this stage, if they are due to dangling bonds or to impurities (e.g. metal impurities or oxygen complexes). In addition, the observed Fermi level shift (with increasing dilution) may also fill up defect states with electrons, making them inactive for electron trapping (and invisible for TOF) even though they remain present. Thus, we cannot exclude the presence of such states in highly diluted materials on the basis of our TOF measurements. This also means, that our observation of important trap state density in low-dilution material is not necessarily contradictory to the optical measurements by CPM, which indicate a higher density of deep defect states for high-dilution material. CPM data reflects the optical transitions from gap states below the Fermi level (occupied by electrons), hence, CPM sees different defect states than post-transient TOF.

\section{CONCLUSIONS}

We have shown that the optical and electrical properties of $\mu \mathrm{c}-\mathrm{Si}: \mathrm{H}$ prepared by glow discharge can strongly be influenced by changing the dilution ratio of silane to hydrogen. 
Thereby, not only structural changes are involved, but also an enhanced incorporation of impurities at low deposition rates (typical for high dilution materials), leading to deposition of material with an extrinsic character.

Spectral dependence of the optical absorption coefficient reflects the material changes that occur due to an increased dilution: it directly shows the transition from amorphous to microcrystalline material and also reflects residual amorphous fractions. In the subbandgap region absorption measurements are sensitive to defects and impurities. To extract more quantitative data, more has to be known about the influence of oxygen and on the distortion of absorption spectra due to light scattering.

Time of Flight measurements on the $\mu \mathrm{c}-\mathrm{Si}: \mathrm{H}$ samples show a strongly increased drift mobility of holes when compared to typical a-Si:H. The measured values increase with higher dilution ratios from 1 to $3 \mathrm{~cm}^{2} / \mathrm{Vs}$ for electrons and from 0.4 to $1.2 \mathrm{~cm}^{2} / \mathrm{Vs}$ for holes. The ratio between electron and hole mobilities observed at room temperature is about 2 and is close to that of crystalline silicon; this clearly indicates a transport mechanism which is different from the multiple trapping transport found in a-Si:H.

By using the post-transient TOF technique we have also found in low-dilution material an efficient type of deep electron trap in the middle of the gap whose origin is not yet clear. Further studies are necessary in order to clarify if these traps are due to dangling bonds or to impurities in the material. Furthermore, the Fermi level shift which accompanies the dilution effect, can render "invisible" certain defect states for TOF by filling them up with electrons; thus a direct interpretation of the results becomes difficult.

\section{ACKNOWLEDGMENTS}

This work was supported by the Swiss National Science Foundation under grants FN-39377 and FN-45696.95 and by the Swiss Federal Office of Energy under Research grant EF-REN (93)032.

\section{REFERENCES}

[1] J. Meier, S. Dubail, R. Flückiger, D. Fischer, H. Keppner and A. Shah, Proc. of the 1st WCPVEC, Hawaii, 405 (1994).

[2] N. Beck, J. Meier, J. Fric, Z. Remes, A. Poruba, R. Flückiger, J. Pohl, A. Shah and M. Vaněček, J. Non-Cryst. Sol. 198-200, 903 (1996).

[3] U. Kroll, J. Meier, A. Shah, S. Mikhailov and J. Weber, J. Appl. Phys. 80, 4971 (1996).

[4] J. Meier, P. Torres, R. Platz, S. Dubail, U. Kroll, J.A. Anna Selvan, N. Pellaton Vaucher, C. Hof, D. Fischer, H. Keppner, A. Shah, K.-D. Ufert, P. Giannoulès, J. Koehler, to be published in Mater. Res. Soc. Symp. Proc. (San Francicso, 1996).

[5] M. Goerlitzer, N. Beck, P. Torres, J. Meier, N. Wyrsch and A. Shah, J. Appl. Phys. 80, 5111 (1996).

[6] P. Torres, J. Meier, R. Flückiger, U. Kroll, J.A. Anna Selvan, H. Keppner, A. Shah, S.D. Littelwood, I.E. Kelly and P. Giannoulès, Appl. Phys. Lett. 69, 1373 (1996).

[7] N. Wyrsch, M. Goerlitzer, N. Beck, J. Meier and A. Shah, to be published in Mater. Res. Soc. Symp. Proc. (San Francicso, 1996).

[8] M. A. Green, M. J. Keevers, Progress in Photovoltaics: Research and Applications 3, 189 (1995).

[9] M. Vaněček, D. Cervinka, M. Favre, H. Curtins and A. Shah, Mater. Res. Soc. Symp. Proc. 192, 639 (1990).

[10] J. Y. W. Seto, J. Appl. Phys. 46, 5247 (1975).

[11] G. F. Seynhave, R.P. Barclay, G.J. Adriaenssens, J.M. Marshall, Phys. Rev. B39, 10196 (1989).

[12] J. Kočka, M. Vaněček, P. Macháček, A. Fejfar, E. S̆ípek, Ho-The-Ha, I. Pelant, J. Fric, J. Rosa, Z. Remes, A. Poruba, M. Konagai and W. Kusian, Proc. of the 1st WCPVEC, Hawaii, 437 (1994). 